\title{
STEP-INVARIANT TRANSFORM FROM Z- TO S-DOMAIN A General Framework
}

\author{
JÓZSEF G. NÉMETH and ISTVÁN KOLLÁR \\ DEPARTMENT OF MEASUREMENT AND INFORMATION SYSTEMS \\ BUDAPEST UNIVERSITY OF TECHNOLOGY AND ECONOMICS \\ BUDAPEST, HUNGARY, H-1521 \\ E-MAILS: \{NEMETH,KOLLAR $\} @$ MIT.BME.HU \\ WWW: HTTP://WWW.MIT.BME.HU/\{ NEMETH, KOLLAR $\}$ \\ PHONE: +36 1 463-2057, FAX: +36 1463-4112
}

\begin{abstract}
The conversion from discrete-time to continuous-time models is sometimes quite troublesome. This paper answers related pending questions concerning z-domain poles at the origin, and relating them to s-domain models with time delay. Special attention is devoted to the tradeoff between fractional delay and direct feed-through. The paper places these questions into a general framework of model mapping between z-domain transfer functions and $s$-domain transfer functions, maybe with input delay.

An algorithm to convert $z$-domain models with a pole at the origin is explained. A MATLAB routine has been implemented. The routine allows to execute the conversion either by defining the delay or the allowable direct feed-through. This routine is made available through the $W W W$.
\end{abstract}

Keywords - MATLAB, ZOH transform, discrete-tocontinuous transform, d2c, step-invariant transform, delay system, fractional delay.

\section{Introduction}

When the transfer function of a system was identified in the $z$-domain (sometimes the nature of data requires this, see [1]), it may need to be transformed to the $s$-domain, e. g. to give physical interpretation of the results. However, while transform from $s$-domain to $z$ domain is unique, the other direction is sometimes am- biguous, sometimes there is even no $s$-domain equivalent in the desired model set.

The well-known case is the presence of negative real poles in the $z$-domain model. In [2] an extra manipulation was suggested to overcome the problem. By adding a canceling pole/zero pair to the $z$-domain model the transformation becomes possible, but then ambiguity appears. The undetermined part in the impulse response of the resulting $s$-domain system, as it was shown, is a signal with zero crossings exactly at the sampling instants. These hidden oscillations can be eliminated if we apply a 'parsimony' principle, by which the solution is unique.

Based on these results, in this paper we seek a general framework for model conversion, by treating other cases where waveforms are involved, which are hidden from sampling. We pay special attention to systems with fractional delay.

The natural solution would be to seek transformation between 'proper' transfer functions, that is, between ratios of polynomials in $z$-domain and $s$-domain. With the solution of [2], almost all cases are treated, except $z$-domain systems with poles at the origin. In this case, a delay must appear in the $s$-domain. If we can determine the associated models, we have $s$-domain counterparts of all $z$ domain transfer function models.

$$
\frac{N(z)}{D(z)} \Leftrightarrow e^{-s T} \frac{N(s)}{D(s)}
$$

The approaches and algorithms presented here also fill the gap among the routines in MATLAB, establishing the

\footnotetext{
${ }^{1}$ This work is supported by the Belgian National Fund for Scientific Research, the Flemish government (GOA-IMMI, and BIL99/18), and the Belgian government as a part of the Belgian programme on Interuniversity Poles of attraction (IUAP4/2) initiated by the Belgian State, Prime Minister's Office, Science Policy Programming.
} 
symmetry of conversions (presently $s$-domain models with arbitrary time delay can be transformed to $z$-domain, but the results cannot be transformed back if the delay is not an integer multiple of the sampling interval, see the routines $\mathrm{c} 2 \mathrm{~d}$ and $\mathrm{d} 2 \mathrm{c}$ ). As a second benefit, they give further insight into a general and inherent ambivalence: the behavior of the reconstructed continuos-time model can be determined between sampling instants only by making restrictions in the model class and/or giving extra parameters or rules for the conversion.

First, we expose the theoretical problem of inverting back the $\mathrm{ZOH}$ transform of continuous-time systems, with special care to the case of fractional delay. We discuss a numerical algorithm presented in [4] that addresses this topic.

Then, we are going to use the decomposition into partial fractions and present a new algorithm for the conversion.

Last, we draw the attention to problems emerging with measured data and ensuring parametric models.

\section{Preliminaries}

Identification of continuous-time linear systems can be done either in time domain or in frequency domain. Timedomain methods usually yield a discrete-time model of the system, while frequency domain methods can identify either a discrete-time ( $z$-domain) or a continuous-time ( $s$-domain) model. In order to achieve accurate results, as in evaluating models from measurements, the experiment set-up must meet the requirements prescribed by the applied estimation method [1].

Therefore, it may occur that the consequence of the measurement arrangement is that we estimate a $z$-domain model which then needs to be mapped to the $s$-domain. The $\mathrm{ZOH}$ transform provides such a mapping between models of the two domains, assuming that data samples of the excitation are input to a zero-order-hold term, and the output signal is sampled without extra band-limiting $[1,3]$ :

$$
H(z)=\operatorname{ZOH}\{H(s)\}=\left(1-z^{-1}\right) Z\left\{\operatorname{Sampling}\left\{L^{-1}\left\{\frac{H(s)}{s}\right\}\right\}\right\}
$$

The inverted Laplace transform in the second inner bracelet is the step response of the system, which defines the values at the sampling instants for the resulting $z$ domain system (Sampling \{.\}). Hence, Eq. 1. is also called step-invariant transform and the equation can be rearranged to show the invariance of the step-response in sampling instants:
$Z^{-1}\left\{\frac{z}{z-1} H_{\mathrm{zOH}}(z)\right\}=\operatorname{Sampling}\left\{L^{-1}\left\{\frac{H(s)}{s}\right\}\right\}$

Given a sampling rate, all transformations involved in Eq. 1-2. are well-defined, thus the conversion exists and is unique for all transfer functions in the $s$-domain. In particular, poles can be matched as $z_{i}=e^{p_{i} T}$, where $z_{i}$ and $p_{i}$ are the $i^{\text {th }}$ pole in the $z$-domain and $s$-domain, respectively.

However, converting from the $z$-plane to the $s$-plane may cause problems, because interpolation which is the invert operation to sampling, can only be carried out properly if some extra rule is given: e.g., for band-limited signals the Nyquist-criterion is met (this is certainly not the case since the step response is never band-limited). There are at least two cases, when this latter criterion is strongly violated, causing the following problems:

1. When the $z$-domain model contains a negative real pole, the complex logarithm of this pole is neither unique, nor leads to real coefficients in the $s$-domain transfer function [2]:

$$
p_{i}=\frac{1}{T}\left(\ln z_{i}+j 2 \pi m\right), \quad \forall m \in N
$$

2. If the $z$-domain model (in $z$ ) has pole(s) in the origin, the model contains pure time-delay. Yet, the amount of delay cannot be determined unambiguously. (In fact, the exponential coefficient in the $s$-domain transfer function representing the delay can be expanded into an infinite series leading to an infinite number of poles, with the position not restricted. This indicates that the Nyquist criterion is going to be violated.)

As it was shown in [2], adding a canceling pole-zero pair to the $z$-domain model can circumvent the first problem (although leads to ambiguity).

As far as the second problem is concerned, [3] explains a straightforward way to convert $s$-domain transfer functions with fractional time-delay into the $z$-domain, whereas [4] gives a simple MATLAB code for conversions in both directions. However, the conversion 'from $z$ to $s$ ' relies on non-linear, zero-order approximation, and the article does not treat the possibility of direct feed-through, we will treat later. Instead, it excludes the possibility of direct feed-through from the input to the output of the system. In the case of a real-world system, this possibility should not be a priory excluded.

\section{Illustration of the problem of a delay}

To illustrate the problem of systems with time delay, let us consider the following example: 


$$
H_{1}(s)=e^{-0.2 s T} \frac{4 s+5}{s^{2}+2+3}
$$

is the transfer function of a continuous time system with a time delay of $0.2 T$, where $T$ is going to be the sampling period and $T=1 \mathrm{~s}$. We can calculate the $\mathrm{ZOH}$ equivalent for the sampled system (this can be done e.g. by the command $\mathrm{c} 2 \mathrm{~d}$ in MATLAB):

$$
H_{2}(z)=\frac{2.019 z^{2}-0.2029 z-0.1151}{z^{3}-0.1147 z^{2}+0.1353 z}
$$

If we factor out $z$ from the denominator as a unit delay, then the resulting expression will contain an improper fraction (a direct feed-through).

$$
H_{2}(z)=z^{-1} \frac{2.019 z^{2}-0.2029 z-0.1151}{z^{2}-0.1147 z+0.1353}
$$

This improper fraction in Eq. $4 \mathrm{c}$ can be interpreted in two radically different ways:

- either we consider the integer part as a direct feedthrough from the system input onto its output, multiplied by $z^{-1}$ (leading to the model in Eq. 4d)

- or we can account for it as a negative fractional time delay in the continuous-time model (suppressing the direct feed-through in that domain). In this latter case the integer delay $z^{-1}$ and the negative fractional delay results in a positive fractional delay (leading back to the original model in Eq. 4a.)

As a third possibility, we can combine the two interpretations in a trade-off, thus getting infinite number of $s$ domain models for a single transfer function in $z$.

MATLAB's d2c routine cuts the question short, by simply not letting to convert poles near to the origin. Therefore, we may only choose to eliminate the pole in 0 from $H_{2}(z)$, transform it to the $s$-domain, then complement it with the unit delay that we previously omitted:

$$
H_{3}(s)=e^{-s T} \frac{2.019 s^{2}+0.59 s+5}{s^{2}+2 s+3}
$$

Thus we got two systems $\left(H_{l}(s)\right.$ and $\left.H_{3}(s)\right)$ which are different, even though they produce exactly the same values at the sampling instants. Although even more complicated cases exist, this illustrates the general problem. Namely, at sampling instants the different transforms produce the same values, while inside the intervals between samples the outputs differ. This inter-sample behavior can be of different nature. In the case of poles at the Nyquist frequency band-limit, the difference takes the form of hidden oscillations, while by trading the amount of the fractional delay vs. the direct feedthrough term, the difference can be concentrated in the first sampling interval of the step response.
In Figs. 1-3 the step responses, the impulse responses, and the Bode diagrams are depicted, respectively, for the

transfer functions $H_{1}(s)$ and $H_{3}(s)$. After the first sampling instant, the output is identical for the step and the impulse responses even between the samples. This is, of course, not the case for a general piece-wise constant excitation, which can be represented as a weighted sum of shifted step functions. In the general case the input changes from sample to sample and the responses of the two systems are different between samples at any instant.

The Bode diagrams illustrate the consequences of this difference. At higher frequencies the excitation contains greater 'steps', and the difference is more stressed. The amplitude characteristic of the system $\mathrm{H}_{3}(s)$ converges to the amount of the direct-feed-through, while that of $H_{1}(s)$ rolls off above the pole frequency $\omega_{\mathrm{P}}$. (The sampling radian frequency is $\omega_{\mathrm{S}}=6.28 \mathrm{rad} / \mathrm{s}$.)

The exponentially decreasing curves in the log-lin phase plots correspond to the linear phase shift introduced by the delay.

Clearly, the greater the difference in the delay of the two systems and greater the damping, the greater is the difference in the frequency behavior. Increasing the sampling rate can reduce the uncertainty of the delay and the difference in the Bode plots.

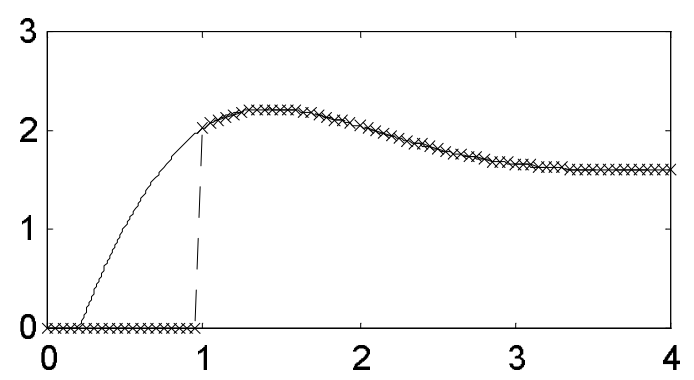

Fig. 1 Step response of $H_{1}(s)$ (continuous line) and $H_{3}(s)$ (crosses and the Dirac delta)

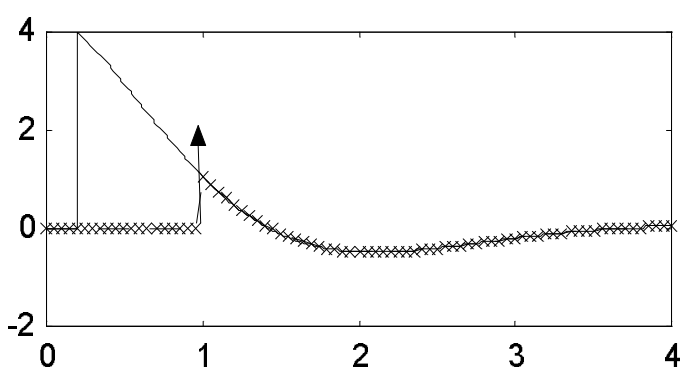

Fig. 2 Impulse response of $H_{1}(s)$ and $H_{3}(s)$ 


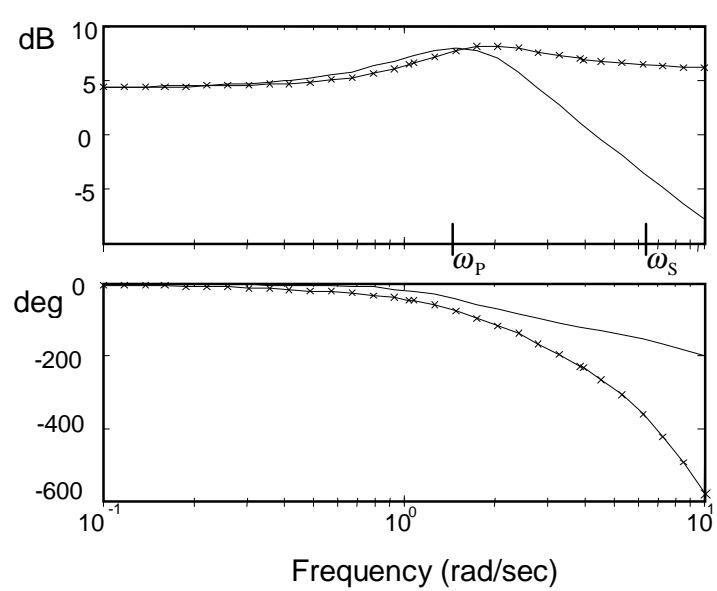

Fig. 3 Bode diagrams of $H_{1}(s) \& H_{3}(s)$

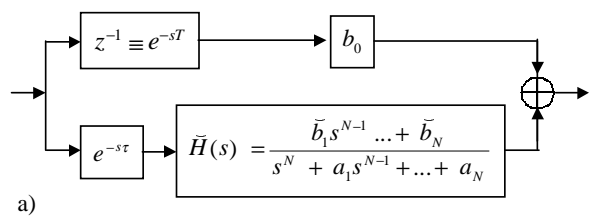

a)
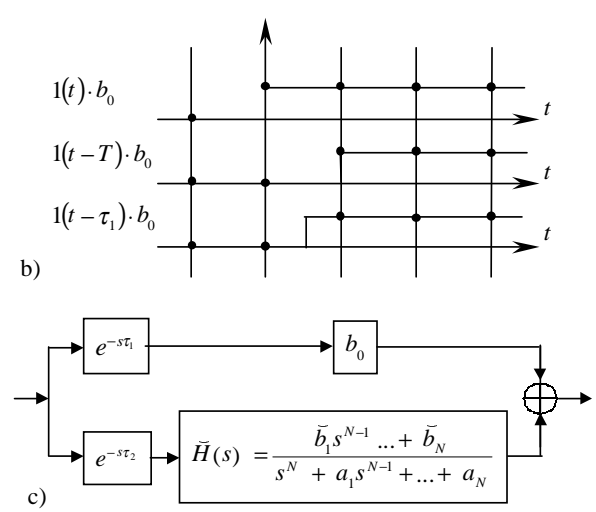

Fig. 4 a) Systems considered initially;

b) Ambivalence of the delay for the direct term (step responses: without delay, with fractional delay, and integer delay)

c) Class of systems that could be modelled exploiting the ambivalence

\section{Consequences}

So far we have considered two extremes: a dynamic system containing a fractional time-delay and an integer delay term followed by a system with direct feed-through, i.e. a dynamic system in parallel with a static gain. We may argue for the first case, but may not simply exclude the second one. Furthermore, there is the odd idea to combine these two solutions (Fig. 4a).

There is, however, a further case of ambivalence: since the excitation is piece-wise constant, the delay of the direct feed-through term is only determined up to the sam- pling period. In other words, the fact that the step function is constant after 0, causes another ambivalence (Fig 4b).

By taking this into account, the class of systems included in the model set can be quite wide (Fig. 4c). A reasonable interpretation is when the delay is the same for the two branches (Eq.5b). This restriction also ensures uniqueness if either $b_{0}$ or $\tau$ is given as parameters to the mapping (see later). is:

Revisiting the framework, the class of system models

$$
H(z)=\frac{d_{0} z^{N_{d z}}+d_{1} z^{N_{d z}-1} \ldots+d_{N_{d z}}}{z^{N_{c z}}+c_{1} z^{N_{c z}-1}+\ldots+c_{N_{c z}}}
$$

where $d_{i} \in \mathrm{R}, c_{i} \in \mathrm{R}$,

$$
H(s)=e^{-s \tau} \frac{b_{0} s^{N_{b s}}+b_{1} s^{N_{b s}-1} \ldots+b_{N_{b s}}}{s^{N_{a s}}+a_{1} s^{N_{a s}-1}+\ldots+a_{N_{a s}}}
$$

where $b_{i} \in \mathrm{R}, a_{i} \in \mathrm{R}, 0<\tau$ and the poles are inside the Nyquist band. (This resolves the ambiguity coming from hidden oscillations; the technique described in [2] can be applied if necessary.)

We will assume that the order of the denominator is finite, therefore the poles are necessarily given as the complex logarithms of the $z$-domain poles. This statement is necessary because the curve interpolating the samples of the $z$-domain step-response is only valid after the entering point of the signal described by the rational fraction part of the $s$-domain function. If a fractional delay is allowed, then extrapolation can only be made for the sample interval before the first non-zero sample in the step-response. This extrapolation will be unique if we consider an interpolation (respecting the Nyquist band) with an additional point instead of the zero. This additional information is assumed to be specified if we make the restriction for the poles.

For the sake of simplicity, in most of the discussion, we consider that the integer part of fractional delays can be treated separately. However, in the next section we are going to show that this is a further restriction, which is a possible option to avoid further ambiguities.

\section{Further ambiguities}

In fact, by omitting the fractional delay in Eq. 4a, the ambivalence still exists, because the transfer function obtained by mapping can always be extended by a canceling pole-zero pair in the origin, and we can factor out from the denominator an integer delay. As a result we get a system containing an integer delay and a direct feedthrough in the rational fraction part of the transfer function (Fig. 5). 


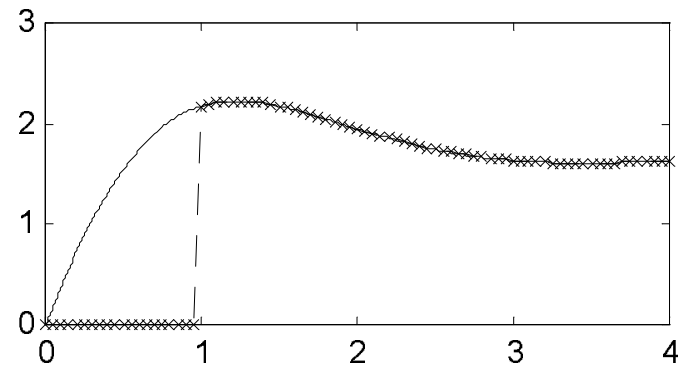

Fig. 5 Step responses of a system with no delay and its ' $\mathrm{ZOH}$ alias' containing a direct feed-through in the rational fraction part of the transfer function.

All the more, even if a direct feed-through is not allowed in the rational transfer function by our definition of causality, we can meet ambiguity. Simply consider the case when we modify Eq. 4b so that only the first-order term of the numerator is different from zero, and we simplify by $z$ (Eq. 6).

$$
\begin{aligned}
H_{2 \mathrm{~m}}(z) & =\frac{-0.2029 z}{z^{3}-0.1147 z^{2}+0.1353 z} \\
& =\frac{-0.2029}{z^{2}-0.1147 z+0.1353}
\end{aligned}
$$

In this case, the interpolation curve of the stepresponse has a zero-crossing at the sampling instant 1 . Thus, depending on whether we factor out a delay (by multiplying by $\mathrm{z} / \mathrm{z}$ ) or not, we can get through mapping to two different continuous-time systems containing no fractional delay and no direct feed-through (Fig. 6).

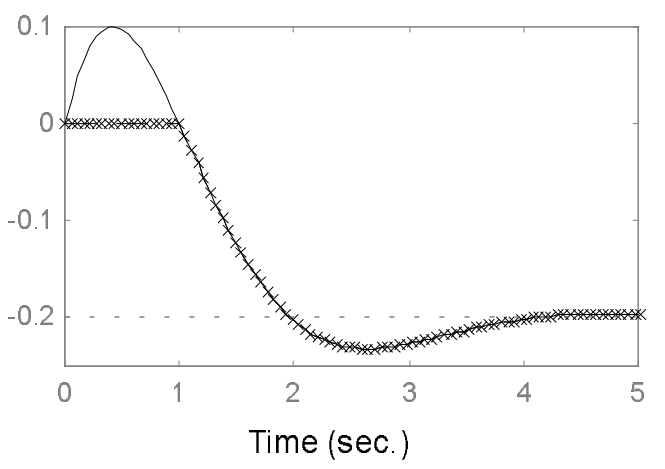

Fig. 6 Step responses of a system with no delay and its ' $\mathrm{ZOH}$ alias' containing no direct feed-through.

\section{$\underline{\text { V. Solution by partial fractions }}$}

Considering the $s$-to- $z$ transform of a system with fractional delay, it is straightforward that if a $z$-domain model was obtained by ZOH-transform, its $s$-domain counterpart must exist. The minimum goal can be to find this path, and add it to MATLAB's d2c. However we must count with ambiguity, and we should be aware of all the possible $s$-domain models. Furthermore, we should be able to determine whether a given $z$-domain system could be obtained from an $s$-domain system by $\mathrm{ZOH}$-transform, or not.

Decomposition of the $z$-domain model into partial fractions, and transform term by term is the simplest way to break down the problem into the transformation of small building blocks. Since the step response is zero before $t=0$, the delay must be decomposed into an integer delay and a negative fractional delay yielding a noncausal system ([3]):

$e^{-\lambda T s}=e^{-l T s} e^{m T s}$, where $\lambda>0, l \in \mathrm{N}^{+}$and $0<m<1$

Here we only consider the case when $l=1$, hence $0<\lambda<1$, because in the general case the remaining integer delay can be treated separately.

After separating the integer delay $e^{-T s}$ the pre-shifted step response must be transformed. (By using polynomials in $z^{-1}$, the fraction becomes proper.) Single poles can be transformed as:

$$
e^{m T s} \frac{r_{i}}{s-s_{i}} \rightarrow r_{i} e^{s_{i}(k+m) T} \rightarrow \frac{e^{m T s_{i}} r_{i}}{1-z^{-1} z_{i}}
$$

Double poles can be transformed with a bit more care:

$$
\begin{aligned}
e^{m T s}( & \left.\frac{r_{i 1}}{\left(s-s_{i}\right)}+\frac{r_{i 2}}{\left(s-s_{i}\right)^{2}}\right) \rightarrow r_{i 1} e^{s_{i}(k+m) T}+r_{i 2}(k+m) T e^{s_{i}(k+m) T} \\
& \rightarrow\left(r_{i 1}+r_{i 2}(m-1) T\right) e^{s_{i}(k+m) T}+r_{i 2}(k+1) T e^{s_{i}(k+m) T} \\
& \rightarrow \frac{\left(r_{i 1}+r_{i 2}(m-1) T\right) e^{m T s_{i}}}{1-z^{-1} z_{i}}+\frac{T z_{i} e^{m T s_{i}} r_{i 2}}{\left(1-z^{-1} z_{i}\right)^{2}}
\end{aligned}
$$

Terms containing poles of higher multiplicity can be handled in a similar way, so we do not discuss it here.

For single poles:

$$
r_{i}=\frac{b\left(s_{i}\right)}{a^{\prime}\left(s_{i}\right)} \text { and } R_{i}=r_{i} e^{m T s_{i}}
$$

where $b\left(s_{i}\right)$ and $a^{\prime}\left(s_{i}\right)$ are the numerator and the derivative of the denominator of the step response at $s_{i}$, respectively. $R_{i}$ denotes the residual of the corresponding term in the discrete transfer function (in $z^{-1}$ ) comprising the delay. For double poles:

$$
r_{i 1}=\frac{b^{\prime}\left(s_{i}\right)-\frac{b\left(s_{i}\right)}{a^{\prime \prime}\left(s_{i}\right)} a^{\prime \prime \prime}\left(s_{i}\right)}{a^{\prime \prime}\left(s_{i}\right) / 2} \text { and } r_{i 2}=\frac{b\left(s_{i}\right)}{a^{\prime \prime}\left(s_{i}\right) / 2}
$$




$$
R_{i 1}=\left(r_{i 1}+r_{i 2}(m-1) T\right) e^{m T s_{i}} \text { and } R_{i 2}=T z_{i} r_{i 2} e^{m T s_{i}}
$$

All the equations are linear in the coefficients of $b$, so they can be solved by standard methods. The only parameter which needs to be sought by nonlinear optimization is $m$.

\section{Numerical results by partial fractions}

The algorithm based on partial fractions is available in [7]. It gives very good accuracy, even with higherorder models and double real poles. With double complex poles, however, the accuracy dropped significantly. In general, when there are many poles, the starting of the step response is very smooth. Thus identifying the delay is more difficult on the one hand, but the relevance of the question diminishes on the other hand (except the case when the delay is the parameter to be retrieved, but then special techniques might be applied.)

The algorithm handles complex poles the same way as real poles. Numerical stability can be significantly improved by unifying conjugate poles into real valued resonator terms.

As another way for improving numerical stability of the algorithm is division of the rows of the equations by the right-hand sides of the equations. By this, we can get a least squares estimator with balanced weights. It is even possible that other weightings are more advantageous.

\section{Application to identification}

In practical problems the models come from measured data, and the following questions need to be tackled:

- Is the excitation (with good approximation) piecewise constant? Is the system linear with good approximation?

- What can be considered as zero? (One will never get poles exactly at zero, or coinciding roots, or canceling pole-zero pairs. A model of the uncertainty should be included.)

'No' to the first group of questions may result in bias.

The second group of questions should be considered by taking into account confidence bounds. For instance, if a confidence bound overlaps the origin, then the pole may be considered as equal to zero. However, since a pole at zero leads to a structurally different model, a related question is which is the 'proper' model for a given system, and which choice is justified by the data.

More complex questions are how confidence bounds (or other measures of uncertainty) can be transformed along with model parameters. This becomes necessary once we want to compare models of continuous-time systems obtained by $s$-domain and $z$-domain methods.

\section{Conclusions}

Model mapping from the $z$-domain to the $s$-domain has been studied. Ambiguous cases have been pointed out. The common element in these cases is the presence of a delay in the s-domain step-response (or direct feedthrough with one-step delay).

For physical reasons we cannot exclude the possibility of systems with direct feed-through from the set of causal systems. As it has been demonstrated: direct feed-through combined with any kind of continuous-time delay can be mistaken for fractional delay. The differences between resulting s-domain models can be quite important even in simple cases.

We gave an algorithm to convert $z$-domain models containing a pole in zero (case not supported by $\mathrm{d} 2 \mathrm{c}$ in MATLAB), which also allows a direct feed-through in the rational part of the $s$-domain transfer function.

Questions which still have to be answered:

- Does every $z$-domain transfer function have a corresponding $s$-domain equivalent or not?

- $\quad$ Are there any more ambiguities than enumerated in this paper? Is the transform unambiguous when direct feed-through is excluded?

- What is the consequence of noisy measurements, that is, uncertain $z$-domain models? How can we decide if a coefficient is zero or not?

\section{References}

[1] Schoukens, J., R. Pintelon and H. Van hamme, "Identification of Linear Dynamic Systems using Piecewise Constant Excitations: Use, Misuse and Alternatives", Automatica, Vol. 30, No. 7, 1994, pp. 1153-69.

[2] Kollár I., G. Franklin and Rik Pintelon, "On the equivalence of z-domain and s-domain models in system identification", Proc. IEEE Instrumentation and Measurement Technology Conference, Brussels, Belgium, June 4-6, 1996. pp. 14-19.

[3] Franklin, G. F., J. D. Powell and M. L. Workman, Digital control of dynamic systems. Reading, MA: AddisonWesley, 2. ed., 1992.

[4] Kuznetsov, A. G. and D. W. Clarke, "Simple numerical algorithms for continuous-to-discrete and discrete-tocontinuous conversion of the systems with time delay", SYSID'94, 10th IFAC Symposium on System Identification, Copenhagen, July 4-6, 1994, Vol. 3., pp. 633-638.

[5] Evans, C., D. Rees, L. Jones and D. Hill, "Time and frequency domain identification of jet engine dynamics: Problems and solutions", SYSID'94, 10th IFAC Symposium on System Identification, Copenhagen, July 4-6, 1994, Vol. 2., pp. 243-48.

[6] Golub, G. and C. F. Van Loan, Matrix computations, Johns Hopkins University Press, 1989.

[7] http://www.mit.bme.hu/ nemeth/z2s/ 